\title{
TRATAMENTO DO MEGAESÔFAGO CHAGÁSICO GRAU II POR LAPAROSCOPIA : EXPERIÊNCIA EM 12 CASOS
}

\section{LAPAROSCOPIC MANAGEMENT OF ESOPHAGEALACHALASIA: EXPERIENCE IN 12 CASES}

\author{
Antonio Carlos Valezi, TCBC-PR ${ }^{1}$ \\ Jorge Mali Júnior, ${ }^{2}$ \\ Antonio César Marson ${ }^{3}$ \\ Edivaldo Macedo de Brito, ACBC-PR ${ }^{4}$ \\ José Carlos Lacerda de Souza, ACBC-PR ${ }^{4}$
}

\begin{abstract}
RESUMO: Objetivo: Avaliar os resultados da cardiomiotomia com fundoplicatura parcial por vídeolaparoscopia (Heller-Dor) no tratamento de 12 pacientes portadores de megaesôfago grau II. Método: Foram analisados prospectivamente 12 pacientes com megaesôfago não avançado (grau II segundo classificação de Ferreira - Santos ), submetidos a cardiomiotomia à Heller por laparoscopia associado à confecção de vávula anti-refluxo ( fundoplicatura à Dor ), no Hospital Universitário da Universidade Estadual de Londrina, no período de Janeiro de 1999 a Dezembro de 2001. Resultados: Não houve necessidade de conversão para laparotomia em nenhum caso. A pressão média do esfincter inferior do esôfago ( EIE ) no pré-operatório foi de 39,1 $\mathrm{mmHg}$ (normal de 15 a $30 \mathrm{~mm} \mathrm{Hg}$ ), e no pós-operatório (seis meses) de $12,5 \mathrm{mmHg}$. Em relação à sintomatologia pós-operatória, nove dos 12 pacientes ficaram assintomáticos, sendo que três pacientes apresentaram disfagia, sialorréia e pirose, respectivamente. Ocorreram três complicações intra-operatórias: dois casos de perfuração gástrica e um de perfuração esofágica. Não houve mortalidade no intra ou pós-operatório. Conclusões: Podemos concluir, baseado neste estudo, que a cardiomiotomia a Heller associada à fundoplicatura a Dor por vídeo-laparoscopia, após seis meses de acompanhamento, mostrou-se eficaz no tratamento do megaesôfago grau II.
\end{abstract}

Descritores: Acalasia esofágica; Laparoscopia; Fundoplicatura.

\section{INTRODUÇÃO}

O megaesôfago, seja de origem desconhecida ou de etiologia chagásica, caracteriza-se pela ausência ou diminuição acentuada dos plexos nervosos da parede esofágica, ocasionando incoordenação das contrações do corpo esofágico (aperistalse), e abertura ausente, incoordenada ou incompleta do esfíncter inferior do esôfago (acalasia), que resulta em estase esofágica, dilatação progressiva do órgão e diminuição da sua capacidade de contração. A destruição dos plexos nervosos intramurais do esôfago é irreversível, de tal forma que qualquer tratamento conservador será meramente paliativo visando apenas ao alívio dos sintomas ${ }^{1}$.

O tratamento cirúrgico da acalasia foi realizado através de várias técnicas, mas foi com a cardiomiotomia proposta por Gottstein em 1901 e realizada por Heller em 1913, que o tratamento do megaesôfago não avançado teve seus melhores resultados 2,3 .

Baseados na agressão representada pela laparotomia e no fato de que a opção não invasiva

1. Professor Adjunto Doutor do Departamento de Cirurgia da Universidade Estadual de Londrina.

2. Ex-Médico Residente de Cirurgia Geral da Universidade Estadual de Londrina.

3. Professor Assistente Mestre do Departamento de Cirurgia da Universidade Estadual de Londrina.

4. Professor Adjunto Mestre do Departamento de Cirurgia da Universidade Estadual de Londrina.

Recebido em 29/07/2002

Aceito para publicação em 20/04/2004

Trabalho Realizado na Disciplina de Cirurgia do Aparelho Digestivo da Universidade Estadual de Londrina. 
(dilatação da cárdia) apresenta resultados similares e não impede um tratamento cirúrgico no futuro,vários autores contra-indicam a cirurgia como primeira escolha para o megaesôfago não avançado, apesar do mesmo ter resultados melhores e mais duradouros que o tratamento conservador ( dilatação ). Por isso, tradicionalmente, o tratamento cirúrgico do megaesôfago não avançado, tem sido indicado para doentes com disfagia residual após tratamento dilatador ${ }^{4-6}$.

Pretensiosamente, a laparoscopia permitiu a realização do mesmo procedimento que aquele por cirurgia convencional para o megaesôfago não avançado, aliado às vantagens da cirurgia minimamente invasiva ${ }^{7}$.

O objetivo deste estudo foi avaliar os resultados da cardiomiotomia com esofagogastrofundoplicatura parcial por laparoscopia (HELLER-DOR) no tratamento do megaesôfago grau II .

\section{MÉTODO}

Foram analisados prospectivamente 12 pacientes com megaesôfago não avançado (grau II segundo classificação de Ferreira-Santos), submetidos a tratamento cirúrgico - cardiomiotomia à Heller, por laparoscopia, associada à confecção de válvula antirefluxo (fundoplicatura à Dor) - no Hospital Universitário da Universidade Estadual de Londrina, no período de janeiro de 1999 a dezembro de 2001.

Os doentes foram avaliados segundo o sexo, idade, sintomas pré e pós-operatórios, manometria pré e pós-operatória, estudo radiológico contrastado de esôfago, endoscopia digestiva alta (EDA) pré e pósoperatória, tratamentos prévios , complicações do tratamento cirúrgico e resposta à terapêutica através de avaliação clínica do paciente e funcional do esôfago. Os exames realizados no pós-operatório foram feitos seis meses após a cirurgia.

Tabela 1 - Sintomas no pré-operatório.

\begin{tabular}{cc}
\hline SINTOMAS & PACIENTES $(\boldsymbol{\%})$ \\
\hline Disfagia & $12(100)$ \\
Sialorréia & $8(66,6)$ \\
Regurgitação & $7(58,3)$ \\
Pirose & $2(16,6)$ \\
Dor torácica & $1(8,3)$ \\
Odinofagia & $1(8,3)$ \\
Eructação freqüente & $1(8,3)$ \\
\hline
\end{tabular}

A média de idade dos doentes foi de 39 anos (21-67).Oito eram do sexo masculino. Os sintomas pré-operatórios são mostrados na Tabela 1.

Dez doentes apresentavam reação de Machado Guerreiro positiva.

O estudo radiológico contrastado de esôfago mostrou megaesôfago grau II em todos os doentes, segundo classificação de Ferreira Santos.

Dos 12 doentes, cinco haviam sido previamente tratados por dilatação da cárdia.

Um doente apresentava colelitíase à ultrasonografia abdominal.

O preparo pré-operatório dos pacientes incluía jejum de 8 horas, dieta líquida e lavagem do esôfago 24 horas antes da cirurgia.

$\mathrm{O}$ ato operatório iniciava-se com o paciente em posição de litotomia com proclive de 30 graus, sob anestesia geral, com o cirurgião se posicionando entre as pernas do doente. O pneumoperitônio era realizado através de punção com agulha de Veress na região umbilical. O primeiro trocarte era colocado na linha mediana a $20 \mathrm{~cm}$ do apêndice xifóide $(10 \mathrm{~mm}-$ óptica de 30 graus). O segundo trocarte, de $5 \mathrm{~mm}$, subxifóideo, para o afastador de fígado; o terceiro $(5 \mathrm{~mm})$ e o quarto $(10 \mathrm{~mm})$ eram colocados nas regiões subcostal direita e esquerda respectivamente, (pinças de trabalho). O quinto e último trocarte $(10 \mathrm{~mm})$ era colocado no flanco esquerdo (pinça para tração do estômago). Inicialmente afastava-se o fígado e tracionava-se o fundo gástrico. Abria-se o pequeno omento e realizava-se a liberação do esôfago terminal, iniciando-se pelo pilar direito do diafragma, face anterior e pilar esquerdo. Em seguida liberava-se o ligamento gastro-frênico e fazia-se a ligadura dos vasos curtos, quando necessário. Realizava-se a dissecção da face posterior do esôfago, circundando-o com um dreno de Penrose $\mathrm{n}^{\circ} 1$ para tração. A sonda de Fouchet era introduzida e executava-se cardiomiotomia extramucosa, $5 \mathrm{~cm}$ acima e $3 \mathrm{~cm}$ abaixo da transição esofagogástrica. Procedia-se à sutura de $6 \mathrm{~cm}$ entre o fundo gástrico e a face posterior direita do esôfago, e uma segunda linha de sutura, de mesma extensão entre o fundo gástrico e a parede anterior esquerda do esôfago, confeccionando-se uma fundoplicatura anterior de 180 graus, que cobria a área de mucosa esofágica exposta. Nos pacientes com hiato esofagiano alargado (diagnosticado por visão laparoscópica) foi realizada hiatoplastia. Uma paciente foi submetida à colecistectomia no mesmo procedimento. 
Os pacientes foram acompanhados por um período de nove a 39 meses (média de 13.2 meses) e questionados sobre sua sintomatologia.

$\mathrm{Na}$ análise estatística foi utilizado o teste de Wilcoxon, não paramétrico, para comparação das medidas de pressão. A análise das variáveis numéricas foi feita pelo teste de Mann-Withney.

\section{RESULTADOS}

A operação foi completada por via laparoscópica em todos pacientes, sem necessidade de conversão para laparotomia. Uma doente com colecistopatia calculosa foi tratada por colecistectomia laparoscópica associada.

A manometria foi realizada em todos os doentes no pré-operatório e pós-operatório (seis meses após a cirurgia), e mostrou diminuição importante da pressão do esfincter inferior do esôfago (EIE) no pósoperatório em todos os casos. Nove dos doze doentes apresentavam alterações motoras do corpo esofágico ao exame manométrico pré-operatório. A pressão média do EIE no pré-operatório foi de $39,1 \mathrm{mmHg}$ (normal $=15-30 \mathrm{mmHg}$ ), e no pós-operatório de 12,5 mmHg. Os resultados são mostrados no Gráfico 1.

Em relação à sintomatologia pós-operatória, nove dos 12 pacientes ficaram assintomáticos, e três apresentaram disfagia, sialorréia e pirose, respectivamente. Este último foi considerado curado dos sintomas de acalasia, porém com sintomas de refluxo gastro-esofágico (Tabela 2).

Os resultados da endoscopia digestiva alta no pré e pós-operatório são mostrados na Tabela 3.
Tabela 2 - Sintomas pós-operatorios.

\begin{tabular}{cc}
\hline Sintomas & Pacientes \\
\hline Assintomático & 9 \\
Disfagia & 1 \\
Sialorréia & 1 \\
Pirose & 1 \\
\hline
\end{tabular}

Ocorreram três complicações intra-operatórias: dois casos de perfuração gástrica e um de perfuração esofágica, os mesmos identificados e tratados prontamente por rafia e drenagem da cavidade abdominal. Desses três doentes, dois haviam sido submetidos a tratamento prévio com dilatação.

Não houve mortalidade no intra ou pós-operatório nesta série.

\section{DISCUSSÃO}

O tratamento cirúrgico do megaesôfago não avançado através da cardiomiotomia, começou a ser considerado após o advento da dilatação da cárdia sob controle endoscópico, que tornou o procedimento mais seguro e eficaz. Muitos autores argumentaram a favor da dilatação, devido ao baixo índice de complicações, ótima relação custo-benefício, curto tempo de internação e baixa incidência de refluxo gastroesofágico. Em contrapartida a cardiomiotomia isolada pela via convencional apresentava altos índices de refluxo gastroesofágico, custos mais elevados e maior período de recuperação ${ }^{5-7}$.

Gráfico 1 - Manometria do EIE no pré e pós-operatório ( $p<0,004$ para todos os pacientes).

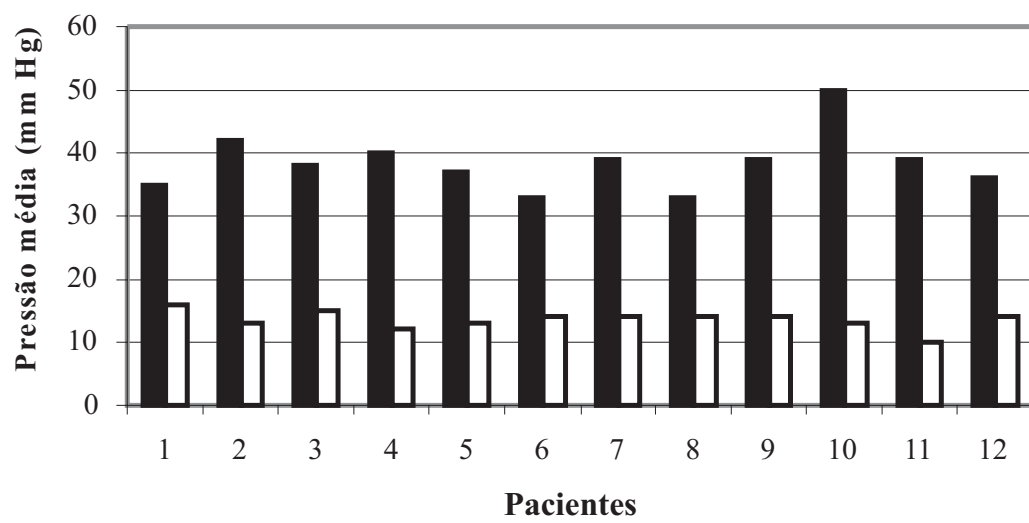

\author{
Pré-operatório \\ $\square$ Pós-operatório
}


Tabela 3 - Endoscopia Digestiva Alta (EDA) no pré e pós-operatório.

\begin{tabular}{c|c|c}
\hline Resultado da EDA & \multicolumn{2}{|c}{ Número de Pacientes } \\
\hline & PRÉ - OPERATÓRIO & PÓS-OPERATÓRIO \\
\hline Normal & 2 & 3 \\
Esofagite & 6 & 2 \\
Gastrite & 8 & 10 \\
\hline
\end{tabular}

A cardiomiotomia por laparoscopia veio resolver tal pendência, a favor da cirurgia, com resultados mais duradouros que as dilatações, associada a curto tempo de internação, baixo índice de complicações e de refluxo gastro-esofágico ${ }^{8}$.

Neste estudo, os doentes com megaesôfago grau II, segundo classificação de Ferreira Santos, sem cirurgia prévia, foram tratados por cardiomiotomia à Heller e fundoplicatura à Dor por via laparoscópica. Os autores tem larga experiência neste tipo de cirurgia pela via laparotômica, uma vez que já a praticam há mais de vinte e cinco anos, e por trabalharem em hospital de referência regional para este tipo de doença.

Alguns autores defendem a não realização de válvula anti-refluxo rotineiramente, justificando que os fatores que levam ao aumento da incidência de refluxo gastro-esofágico no pós-operatório são decorrentes da técnica cirúrgica ( extensão e local da miotomia e também ao maior trauma cirúrgico na cardia) ${ }^{9,10}$. Por outro lado, a maioria dos estudos, indica a confecção de manobra anti-refluxo associada à miotomia, já que esta promove destruição da barreira esfincteriana, permitindo livre refluxo de conteúdo gástrico para o esôfago, com chance de ocorrência de graves esofagites ${ }^{11-13}$. As vantagens de se realizar válvula anti-refluxo são: diminui a incidência de esofagite de refluxo no pós-operatório, propicia mecanismo de proteção da mucosa esofágica contra microperfurações na linha da miotomia, mantém os bordos da miotomia afastados. A taxa de refluxo gastro-esofágico, com a miotomia simples, de até $30 \%$, justifica a associação da fundoplicatura, pela maioria dos cirugiões ${ }^{14}$. As esofagogastrofundoplicaturas laparoscópicas para o tratamento da esofagite de refluxo são hoje amplamente difundidas e existem inúmeras técnicas para sua realização. Utilizamos a fundoplicatura parcial anterior (180 graus) à Dor, pois acreditamos ser de fácil execução técnica, além de não dissecar o esôfago posteriormente e cobrir a mucosa esofágica exposta. A taxa de refluxo gastroesofágico após fundoplicatura à Dor, segundo a literatura é de $10 \%{ }^{9,15}$. Nesta série foi de $8,3 \%$.

Na avaliação clínica pós-operatória, a disfagia, presente em $100 \%$ dos doentes no pré-operatório, persistia em apenas um doente $(8,3 \%)$. A regurgitação presente no pré-operatório em sete doentes, estava ausente no pós-operatório. A pirose, relatada frequentemente no pré-operatório, foi encontrada em um doente no pós-operatório, predizendo assim a eficiente contenção da barreira anti-refluxo. Portanto, no que diz respeito à sintomatologia clínica pré-operatória, em 10 dos 12 doentes ( $83,3 \%$ ) a cirurgia realizada por laparoscopia foi altamente eficaz na abolição dos sintomas, resultado compatível com os obtidos por laparotomia ( $90 \%$ de cura dos sintomas ) ${ }^{16}$.

A endoscopia digestiva alta é etapa fundamental na avaliação do megaesôfago, mesmo tendo seu valor diagnóstico e de estadiamento questionados. Além de afastar a presença de câncer de esôfago, que ocorre tardiamente na evolução do megaesôfago, tem importância no seguimento pós-operatório de esofagite $^{17,18}$. Nesta série, $50 \%$ dos pacientes apresentavam esofagite no pré-operatório e 16,6 \% após a cirurgia.

O estudo radiológico contrastado do esôfago é fundamental no diagnóstico do megaesôfago, apesar de não ser bom parâmetro para avaliar a eficácia do tratamento cirúrgico. Neste trabalho, os 12 pacientes foram submetidos a este exame no pré-operatório, evidenciando o diâmetro do esôfago entre 4-7 cm (grau II, segundo Ferreira Santos ) ${ }^{19}$.

A manometria esofágica, além de ser exame importante para o diagnóstico, é fundamental na avaliação dos casos de insucesso no tratamento cirúrgico. A manometria permite constatação de alterações características do megaesôfago, como aperistalse do corpo esofágico, acalásia da cardia, pressão do EIE maior que $26 \mathrm{mmHg}$ e relaxamento incompleto do mesmo $^{12}$. Há correlação entre a queda da pressão do 
EIE, que normalmente é elevada no megaesôfago, e o sucesso do tratamento cirúrgico por laparotomia ${ }^{20}$. Este fato foi corroborado no presente estudo, em que a queda da pressão média do EIE de $39,1 \mathrm{mmHg}$ no pré-operatório para $12,5 \mathrm{mmHg}$ no pós-operatório associou-se à regressão da sintomatologia pré-operatória $7,15,21,22$.

Dentre as complicações intra-operatórias, as perfurações gástricas e esofágicas são as mais frequentes, durante a realização da miotomia ${ }^{23,24}$. Quando reconhecidas no ato cirúrgico, geralmente são tratados sem grandes dificuldades, e não necessitam de conversão para laparotomia ${ }^{25}$. Nesta série, houve dois casos de perfuração gástrica e um caso de esofágica, que foram tratados por rafia, drenagem da cavidade abdominal. Nos doentes tratados por laparotomia, a incidência destas complicações é menor ${ }^{26,27}$. Este fato se deve principalmemte a perda da sensibilidade tátil durante a realização da miotomia laparoscópica ${ }^{28}$. Das três complicações, dois doentes haviam sido submetidos à dilatação endoscópica prévia. Apesar da dilatação poder dificultar a dissecção periesofágica e alterar os planos anatômicos, não podemos estabelecer relação entre a dilatação pré-operatória e complicação intra - operatória e, a dilatação não parece também comprometer os resultados finais da miotomia ${ }^{29,30}$.

A taxa de conversão para laparotomia na literatura é de 0 a $14 \%{ }^{15,26,27}$. Nesta série não houve necessidade de conversão, nem óbito.

Podemos concluir, baseado neste estudo, que a cardiomiotomia à Heller associada a fundoplicatura à Dor por laparoscopia apresenta baixo índice de complicacões, sendo seu emprego seguro e altamente eficaz no tratamento do megaesôfago chagásico grau II. A cirurgia é perfeitamente exequível por laparoscopia, com resultados semelhantes àqueles da laparotomia, aliado aos benefícios da cirurgia minimamente invasiva .

\begin{abstract}
Background: Various techniques have been used for the surgical treatment of esophageal achalasia, however, the best results for non - advanced esophageal achalasia has been achieved by miotomy. Laparoscopy for non advanced esophageal achalasia have been showing similar results than convencional surgery, with the benefits of a minimally invasive procedure. The aim of this study was to analyze the results of laparoscopy Heller miotomy and Dor fundoplication for non - advanced esophageal achalasia. Methods: Twelve patients with non - advanced esophageal achalasia submitted to laparoscopic Heller miotomy and Dor fundoplication from Jan/99 to Dec/01 were prospectively analised. Results: All surgeries were completed by laparoscopy with no conversion. Nine patients were assymptomatic and tree presented dysphagia, regurgitation and heartburn, respectively. These patients had a median follow-up of 13.2 months (9-39). There were two cases of gastric perfuration and one case of esophageal perfuration but no deaths occured in this series. Conclusions: Based in this study, we could conclude that the Heller miotomy associated to the Dor fundoplication by laparoscopy has a low complication rate. Its use was effective and safe for esophageal achalasia. The surgery is perfectively performed by laparoscopy, with results similar to those of laparotomy, added to the benefits of minimally invasive surgery.
\end{abstract}

Key Words: Esophageal achalasia; Laparoscopic; Fundoplication.

\section{REFERÊNCIAS}

1. Pinotti HW, Domene CE, Cecconello I, et al. "Megaesôfago chagásico". In Coelho JC (eds) - Aparelho digestivo - Clínica e cirurgia, Vol. 1. São Paulo. Medsi, 1996, pp. 201-220.

2. Gottstein G - Technik und klinik der oesophagus kopie. Mitt Grenzgeb Med Chir, 1911, 8:183-186.
3. Heller E, Heller E - Extramukose kardioplastik beim chronischem kardiospasmus mit dilatation des oesophagus. Mitt Grenzgeb Med Chir, 1914, 27:167-171.

4. Reynolds JC, Parkman HP - Achalasia. Gastroenterol Clin North Am, 1989, 18(2):223-255.

5. Richter JE - Achalasia: whether the knife or balloon? Not such a difficult question. Am J Gastroenterol, 1991, 86(7):810-811. 
6. Tack J, Janssens J, Vantrappen G - Non-surgical treatment of achalasia. Hepatogastroenterol, 1991, 38(6):493-496.

7. Domene, CE. Cardiomiectomia com fundoplicatura parcial videolaparoscópica no tratamento do megaesôfago não avançado - avaliação clínica e funcional. Dissertação (Livre-Docência em Cirurgia Gastroenterológica). São Paulo. Universidade de São Paulo, 1996.

8. Kumar V, Shimi SM, Cuschieri A - Does laparoscopic cardiomyotomy require an antireflux procedure? Endoscopy, 1998 ,30(1):8-11.

9. Bonavina L, Nosadini, A, Bardini R, et al. - Primary treatment of esophageal achalasia. Long-term results of myotomy and Dor fundoplication. Arch Surgery, 1992, 127(2):222-227.

10. Robertson GS, Lloyd DM, Wicks AC, et al. Laparoscopic Heller's cardiomyotomy without an antireflux procedure. Br J Surg, 1995, 82(7):957-959.

11. Vara-Thorbeck C, Herrainz R - Esophageal achalasia: laparoscopic HeIler cardiomiotomy. Int Surg, 1995, 80(4):376-379.

12. Vogt D, Curet M, Pitcher D et al. - Successful treatment of esophageal achalasia with laparoscopic HeIIer myotomy and Toupet fundoplication. Am J Surg, 1997, 174(6):709-714.

13. Xynos E, Tzovaras G, Petrakis I, et al. - Laparoscopic Heller's cardiomyotomy and Dor's fundoplication for esophageal achalasia. J Laparoendosc Surg, 1996, 6(4):253-258.

14. Yamamura MS, Gilster JC, Myers BS, et al. Laparoscopic Heller myotomy and anterior fundoplication for achalasia: results in a high degree of patient satisfaction. Arch Surg, 2000, 135(8):902-906.

15. Ancona E, Anselmino M, Zaninotto G, et al. Esophageal achalasia: laparoscopic versus conventional open Heller-Dor operation. Am J Surg, 1995, 170(3):265-270.

16. Pinotti HW, Cecconello, I - Megaesôfago chagásico situação atual de seu tratamento. ABCD Arg Bras Cir Dig, 1991, 6(1):18-21.

17. Semm K - Endoscopic appendectomy. Endoscopy, 1983, 15(2):59-64.

18. Cuschieri A - The spectrum of laparoscopic surgery. World J Surg, 1991, 16(6): 1089-1097.

19. Ferreira-Santos R. Tratamento cirúrgico da aperistalse esofágica (megaesôfago). Dissertação. Ribeirão Preto. Univesidade de São Paulo, 1965.
20. Ellenbogen G. Megaesôfago não avançado. Tratamento pela cardiomiotomia associada a esofagogastrofundopexia. Avaliação clínica, morfológica e funcional dos seus resultados. Dissertação (Doutorado em Cirurgia do Aparelho Digestivo). São Paulo. Universidade de São Paulo, 1979.

21. Castrucci G, Porziella V, Granone PL, et al. - Open versus laparoscopic Heller-Dor procedure for oesophageal achalasia: a comparative functional study. Br J Surg, 1998, 85(2):9-10.

22. Cosentini E, Berlakovich G, Zacherl J, et al. - Achalasia. Results of myotomy and antireflux operation after failed dilatations. Arch Surg, 1997, 132(2):143-147.

23. Shimi S, Nathanson LK, Cuschieri A - Laparoscopic cardiomyotomy for achalasia. J R Coll Surg Edinb, 1991, 36(3):152-154.

24. Elder S, Kunin J, Chouri H, et al. - Safety of laparoscopy cholecystectomy on a teaching service: a prospective trial. Surg Laparosc Endosc, 1996, 6(3):218-220.

25. Medina M - Introduction of two new laparoscopic nooses. Surg Laparosc Endosc, 1996, 6(3):247-249.

26. Morino M, Rebecchi F, Festa V, et al. - Laparoscopic HelIer cardiomyotomy with intraoperative manometry in the management of esophageal achalasia. Int Surg, 1995, 80(4):332-335.

27. Rosati R, Fumagalli U, Bonavina L, et al. - Laparoscopic approach to esophageal achalasia. Am J Surg, 1995, $169(4): 424-427$.

28. Beckingham IJ, Callanan M, Louw JA, et al. Laparoscopic cardiomyotomy for achalasia after failed balloon dilatation. Surg Endosc, 1999, 13(5):493-496

29. Patti MG, Feo CV, Arcerito M, et al. - Effects of previous treatment on results of laparoscopic HelIer myotomy for achatasia. Dig Dis Sci, 1999, 44 (11):2270-2276.

30. Ponce J, Juan M, Garrigues V, et al. - Efficacy and safety of cardiomyotomy patients with achalasia after failure of pneumatic dilatation. Dig Dis Sci, 1999, 44 (11):22772282.

Endereço para correspondência

Antonio Carlos Valezi

Rua Santos 777, apto. 1302.

Centro - Londrina - Pr

86020-021

valezi@conectway.com.br 